\title{
Time-resolved electroluminescence studies of III-nitride ultraviolet photonic-crystal light-emitting diodes
}

\author{
J. Shakya, J. Y. Lin, and H. X. Jiang ${ }^{a)}$ \\ Department of Physics, Kansas State University, Manhattan, Kansas 66506-2601
}

(Received 28 April 2004; accepted 25 June 2004)

\begin{abstract}
Transient responses of III-nitride photonic-crystal (PC) ultraviolet (UV) light-emitting diodes (LEDs) were measured by picosecond time-resolved electroluminescence (EL) spectroscopy. Triangular arrays of PCs with different diameters/periodicities were fabricated on $333 \mathrm{~nm}$ UV LEDs for enhancing light extraction efficiency using electron-beam lithography and inductively coupled-plasma dry etching. With the incorporation of PCs on LEDs, the EL decay time constant $\tau$ decreases systematically with the increase of the etched sidewall area indicating the strong effect of the surface recombination. The surface recombination velocities on the $p$-type epitaxial surface and on the sidewall of etched holes on LEDs were determined to be $1.73 \times 10^{4} \mathrm{~cm} / \mathrm{s}$ and 1.48 $\times 10^{5} \mathrm{~cm} / \mathrm{s}$, respectively. The angular distribution of light emission from LEDs with PCs shows slight narrowing in far-field pattern. Because of the increased transient response along with enhanced light extraction, the incorporation of PCs in UV LEDs provide an effective method to control the modulation speed of UV LEDs, which could be very useful for many applications. () 2004 American Institute of Physics. [DOI: 10.1063/1.1786372]
\end{abstract}

III-nitride blue/ultraviolet (UV) light emitting diodes (LEDs) are useful for many applications including solid-state lighting, compact chemical and biological detection, medical research, and health care. In addition to such diverse applications, high intensity and high speed UV-LEDs could also be used as transceivers for covert non-line-of-sight (NLOS) optical communications. Different schemes such as reducing the size of LEDs to $\mu$-LEDs ${ }^{1}$ and heavily doping the LEDs to reduce the minority carrier lifetimes ${ }^{2}$ have been reported to enhance the operation speed of LEDs, However, such schemes generally reduce the total output power of the LEDS.

Periodic hole arrays are typically etched in a semiconductor to create 2D PCs that forbid certain electromagnetic radiation in the lateral direction creating so called "photonic band gaps" (PBGs) in the plane. 2D PCs enhances the light output from LEDs by extracting lateral guided modes of light in the vertical direction. We have obtained a 20 -fold enhancement of light extraction by $\mathrm{PCs}$ using optical pumping in the nitride material at $475 \mathrm{~nm}^{3}$ Recently, we also reported optical power enhancement under current injection by a factor of 2.45 for $333 \mathrm{~nm}$ UV LEDs, as a result of the PC formation. ${ }^{4,5}$ In this letter, we report the picosecond timeresolved EL studies of the LEDs with 2D PCs having different lattice constants and hole sizes. We also measured the angular distribution of light emission from UV-LEDs with and without PCs.

The incorporation of two-dimensional (2D) photonic crystals (PCs) on III-nitride LEDs has been shown to not only significantly enhance the light output of the LEDs, ${ }^{3-5}$ but also improve the modulation speed of the LEDs. The increase in the modulation speed of LEDs is primarily due to enhanced surface recombination. Photoluminescence, ${ }^{6-8}$ cathodoluminescence, ${ }^{9}$ and time-resolved terahertz spectroscopy ${ }^{10}$ are some of the established methods employed to measure the surface recombination velocity of op-

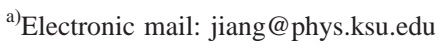

toelectronic materials. Our results from picosecond timeresolved EL spectroscopy on UV PC-LEDs reported in this letter not only reveal a significant increase in the modulation speed of LEDs with PC formation, but also establish time resolved EL as an effective tool to determine the surface recombination velocity of the nitride materials and structures. Moreover, we have also observed a slight narrowing of the far-field emission pattern in PC-LEDs.

The III-nitride UV LEDs structure used were grown by metalorganic chemical vapor deposition (MOCVD) on sapphire substrates. The metalorganic sources used were trimethylgallium for $\mathrm{Ga}$, trimethylaluminum for $\mathrm{Al}$, trimethylindium for $\mathrm{In}$, and ammonia for nitrogen. For $\mathrm{Mg}$ and $\mathrm{Si}$ doping, bis-cyclopentadienyl-magnesium and $\mathrm{SiH}_{4}$ were transported into the reactor chamber during growth. The active region for the LEDs was an $\mathrm{Al}_{0.11} \mathrm{ln}_{0.03} \mathrm{Ga}_{0.86} \mathrm{~N} / \mathrm{Al}_{0.2} \mathrm{In}_{0.03} \mathrm{Ga}_{0.77} \mathrm{~N}$ double quantum wells (DQW). Hexagonal mesa LEDs of side length $120 \mu \mathrm{m}$ was fabricated as described previously. ${ }^{5}$ The PCs with triangular lattice patterns of circular holes with diameters $d=100 \mathrm{~nm}$ to $d=200 \mathrm{~nm}$ and periodicities $a=300 \mathrm{~nm}$ to $a=600 \mathrm{~nm}$ were fabricated using electron-beam (e-beam) lithography and inductively coupled plasma dry etching. AFM image reveals that the depth of the etched holes was around $190 \mathrm{~nm}$ indicating that the holes were etched through into the active layers.

The time-resolved EL setup was a slight modification of the time-resolved photoluminescence PL setup ${ }^{11}$ and has an overall system response of about $30 \mathrm{ps}{ }^{1}$

Figure 1(a) shows a typical EL spectrum of the $333 \mathrm{~nm}$ UV LED. The inset of Fig. 1(a) shows the SEM image of a hexagonal mesa UV LED with triangular lattice holes etched on the mesa to form 2D PCs. Due to the lower magnification of the SEM image, only the interference effect due to PC formation is visible. A higher magnification SEM image of the same PC-LED is shown in Fig. 2(a). The LEDs were driven by the pulse generator with a pulse height of $30 \mathrm{~V}$, width of $0.4 \mathrm{~ns}$ and repetition rate of $1 \mathrm{MHz}$. No change was 


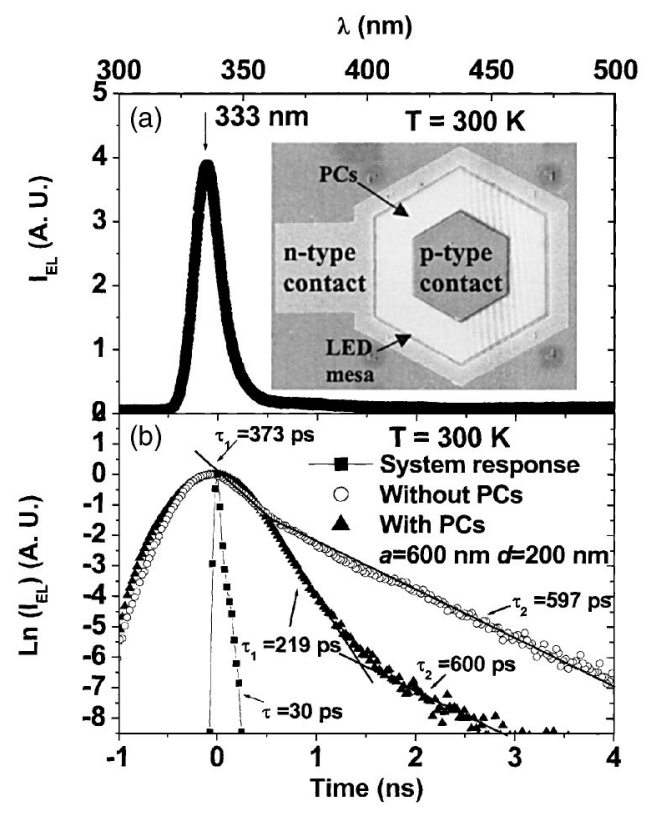

FIG. 1. (a) Typical EL spectrum of the $333 \mathrm{~nm}$ UV LEDs. the inset is the SEM image of LED showing PCs on hexagonal mesa. Hexagonal p-contact layer is at the center of mesa and n-contact layer with a pad surrounds the LED mesa; (b) transient response of the LEDs with PCs $(a=600 \mathrm{~nm}$ and $d=200 \mathrm{~nm})$ and without PCs at the spectral peak wavelength $(\lambda=333 \mathrm{~nm})$. The time-resolved EL setup has the system response $\sim 30 \mathrm{ps}$.

noticed in the peak position and the linewidth of the EL spectrum due to PCs formation, indicating that the spontaneous emission spectrum is not significantly altered by the formation of PCs. The time-resolved EL intensities at the peak wavelength $(\lambda=333 \mathrm{~nm})$ of LEDs with PCs $(a=600 \mathrm{~nm}, d$
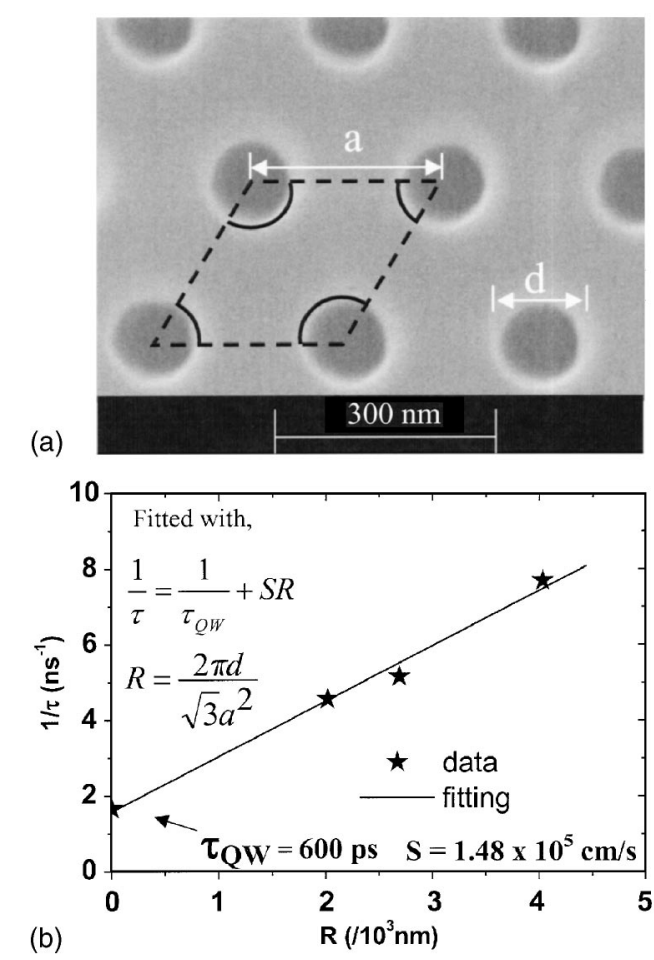

FIG. 2. (a) SEM image of PCs ( $a=300 \mathrm{~nm}$, and $d=100 \mathrm{~nm})$ along with the schematic to calculate the etched sidewall perimeter per unit area, $R$ $=2 \pi d / \sqrt{3} a^{2}$. The dotted parallelogram is the unit area and the curved lines at the four corners are etched perimeters, (b) the EL decay rate $(1 / \tau)$ plotted against $R$. A linear fit of the data yields sidewall surface recombination

velocity $S$ and carrier recombination lifetime $\tau_{\mathrm{QW}}$ in QWs of LED.
Downloaded 30 Mar 2011 to 129.118 .237 .235 . Redistribution subject to AIP license or copyright; see http://apl.aip.org/about/rights_and_permissions
$=200 \mathrm{~nm}$ ) and without PCs are shown in Fig. 1(b). The EL rises fast with a $90 \mathrm{ps}$ time constant for both LEDs. For the LED without PCs, the EL initially decays faster with a time constant $\tau_{1}=373$ ps followed by a slower decay component with a time constant $\tau=597 \mathrm{ps}$, whereas the EL of the LED with PCs $(a=600 \mathrm{~nm}, d=200 \mathrm{~nm})$ decays with a faster time constant $\tau_{1}=214 \mathrm{ps}$ followed by a slower time constant $\tau_{2}$ $\sim 600$ ps.

We believe that the faster decay component in LEDs without PCs is dominated by the surface recombination on the p-type surface. With the incorporation of PC on LEDs, the time constant of the faster decay component $\left(\tau_{1}\right)$ decreases systematically with the increase of etched sidewall area of holes, which indicates that $\left(\tau_{1}\right)$ is controlled by the surface recombination of the carriers on the etched sidewalls. The proximity between the two decay time constants of the slower decay components, $\tau_{2}(=597 \mathrm{ps})$ for LEDs without PCs and the $\tau_{2}(\sim 600 \mathrm{ps})$ for LEDs with PCs, indicates that $600 \mathrm{ps}$ is the carrier recombination lifetime in the QWs. The surface recombination primarily occurs near the junction depletion region, i.e., QW region ${ }^{12}$ and limits the carrier lifetime. ${ }^{8}$ The EL decay rate of LEDs $1 / \tau$ can be expressed as the sum of the carrier recombination rate in QWs and the surface recombination rate:

$$
\frac{1}{\tau}=\frac{1}{\tau_{\mathrm{QW}}}+\frac{1}{\tau_{s}},
$$

where $\tau_{\mathrm{QW}}$ is carrier decay lifetime in QWs and $\tau_{s}$ is recombination lifetime either on the $p$-type surface or on the sidewall surface of the etched holes of PC-LEDs. For the LED without PCs, using the measured values of $\tau_{\mathrm{QW}}=600 \mathrm{ps}$ and $\tau=373 \mathrm{ps}$, Eq. (1) yields a surface recombination lifetime of $\tau_{\mathrm{s}}=985 \mathrm{ps}$ on the $p$-type surface of the LED. Since $\tau_{\mathrm{s}}$ depends upon the area of the surface, a more general parameter, surface recombination velocity $S$, is used to describe the recombination on the surfaces. Considering uniformly distributed charges over the entire p-type region, the surface recombination velocity on the $p$-type surface is given by $S=t / \tau_{s}$, where $(t=0.17 \mathrm{um})$ is the thickness of $p$-type layer. With $\tau_{s}$ $=985 \mathrm{ps}$, the general surface recombination velocity $S$ for p-type GaN surface is thus $1.73 \times 10^{4} \mathrm{~cm} / \mathrm{s}$.

To evaluate the surface recombination of carriers on the etched walls, we consider the geometry of PCs. Figure 2(a) shows a SEM image of PCs $(a=300 \mathrm{~nm}$ and $d=100 \mathrm{~nm})$ along with the schematic to calculate the etched sidewall perimeter per unit area, $R=2 \pi d / \sqrt{3} a^{2}$. Since the depth of the hole is the same for all the PC LEDs, R also represents the sidewall area per unit volume. The LED without PCs has the value of $R=0$. As the product of $\mathrm{S}$ and excess charge density gives the surface recombination rate per unit area, a simple manipulation yields $1 / \tau_{s}=$ SR. With this substitution, Eq. (1) becomes

$$
\frac{1}{\tau}=\frac{1}{\tau_{\mathrm{QW}}}+S R .
$$

The faster component of the EL decay rate $(1 / \tau)$ is plotted against $R$ in Fig. 2(b) Different $R$ values are obtained from LEDs with PCs of different parameters of $a$ and $d .1 / \tau$ increases linearly with $R$ and can be fitted with the Eq. (2) very well. The fit provides the value of $S=1.48 \times 10^{5} \mathrm{~cm} / \mathrm{s}$ and $\tau_{\mathrm{QW}}=600 \mathrm{ps}$. Since the surface recombination mainly dominates the early part of the decay curve in the LEDs, the decay icense or copyright; see http://apl.aip.org/about/rights_and_permissions 
rate $\tau_{2}$, at later times after which most of the excess carriers have diffused away from the surface, represents the carrier decay lifetime in QWs. The values of $\tau_{2}=\tau_{\mathrm{QW}}$ for LEDs with and without PCs further support our conclusion that the measured decay time constant $\tau_{2}$ is the carrier decay lifetime in QWs, $\tau_{\mathrm{QW}}$. The value of $\tau_{\mathrm{QW}}$ measured here for quaternary QW UV LED is longer than the previously reported value of 214 ps for $\operatorname{lnGaN}$ QW blue LED. ${ }^{11}$ This result is consistent with a previous observation that the PL decay lifetime in AlInGaN quaternary alloys is longer than that in $\operatorname{lnGaN}$ ternary alloys. ${ }^{13}$ The surface recombination velocity $S$ has been previously measured for the "as grown" undoped GaN with $S=5 \times 10^{4} \mathrm{~cm} / \mathrm{s}^{14}$ and AlGaN with $S=2.8 \times 10^{4} \mathrm{~cm} / \mathrm{s}^{7}$ Our measured of $S=1.73 \times 10^{4} \mathrm{~cm} / \mathrm{s}$ for P-type GaN surface is comparable to those reported values.

The surface recombination velocity on the "as grown" epitaxial layer is different than that of the etched sidewalls. Etched sidewall has about an order higher value of $S$ as compared to that of the epitaxial surface. Values of $S=8.5$ $\times 10^{5} \mathrm{~cm} / \mathrm{s}$ for epitaxial GaAs (Ref. 10) and $S=6$ $\times 10^{6} \mathrm{~cm} / \mathrm{s}$ for sidewalls of dry-etched GaAs wires at room temperature ${ }^{6}$ also indicate that sidewall recombination dominates in the etched or processed structures. Beside the etchinduced damages, which are thought to be responsible for the increase in $S$ on the etched surface, ${ }^{7}$ sidewall has exposed junction with QW, which increases $S$. Nevertheless, the value of $S$ is more than one order of magnitude smaller in nitride microstructures compared to GaAs based microstructures. This feature makes III-nitrides attractive for the fabrication of photonic structures with reduced dimensions. The internal quantum efficiency of the LED with PCs is expected to decrease slightly due to enhanced surface recombination whereas more light will be extracted from LEDs due to PC formation. Enhancement due to light extraction generally dominates the effect of surface recombination.

The LEDs with higher surface recombination can be modulated at higher speed for many applications. Though there is a trade off between the modulation speed and the optical power enhancement in LEDs with PCs, the unique feature of PC-LEDs is that the maximum modulation speed can be controlled according to specific applications without compromising the optical power output. The highest optical power enhancement we measured was 2.5 -fold $^{5}$ for $333 \mathrm{~nm}$ UV LEDs with PCs $\left(a=600, d=200\right.$, and $\left.R \sim 2 \times 10^{-3} / \mathrm{nm}\right)$. The results shown in Fig. 3(b) indicate that we can achieve a maximum of four-fold enhancement in the modulation speed as a result of PC formation $(a=300, d=100$, and $R \sim 4$ $\times 10^{-3} / \mathrm{nm}$ ). The modulation speed increases with $R$ and, in principle, has no upper limit.

Figure 3 shows the far-field angular distribution of light emission from LEDs with and without PCs. There is a slight narrowing of the far-field pattern of LEDs with PCs. As the periodic structure scatters coherently, increased directionality is expected from LEDs with PCs. Since the etched holes are not perfectly vertical, ${ }^{5}$ the directionality of the emission pattern did not improve significantly. Further improvements in the PCs fabrication, including vertical etching, may give more control over directionality of the emission pattern. With highly directional emission patterns, one may use PCs to replace the LED encapsulation lens, which currently has very

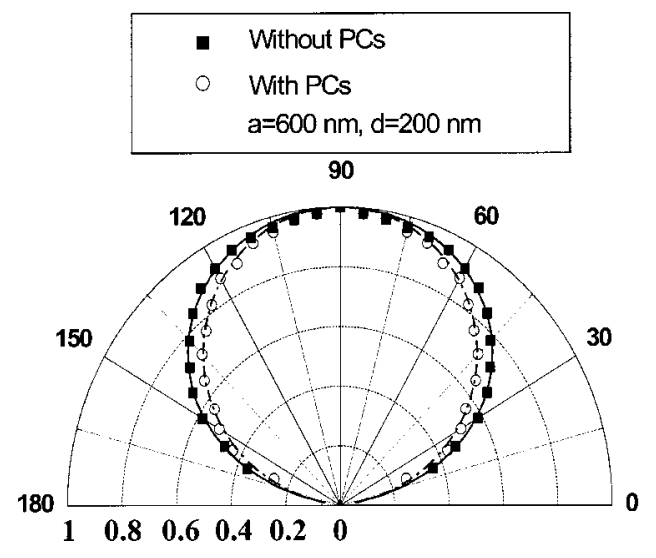

FIG. 3. (a) Far-field angular distribution of light emission from UV-LEDs with PCs $(a=600 \mathrm{~nm}$, and $d=200 \mathrm{~nm})$ and without PCs.

high absorption at deep UV wavelengths $(\lambda<300 \mathrm{~nm})$.

In summary, we have performed the time-resolved (EL) studies of UV-LEDs with and without PCs.The surface recombination velocities as well as the carrier decay lifetime of the LEDs have been obtained. The EL decay time constant $\tau$ changes systematically with the etched sidewall area of the PCs holes. The carrier recombination lifetime $\tau_{\mathrm{QW}}$ of LED is measured to $600 \mathrm{ps}$. The surface recombination velocity $S$ on the etched sidewall of the nitride LED is found to be 1.48 $\times 10^{5} \mathrm{~cm} / \mathrm{s}$, which is about an order of magnitude higher than the value of $S\left(1.73 \times 10^{4} \mathrm{~cm} / \mathrm{s}\right)$ for the epitaxial surface of $\mathrm{p}-\mathrm{GaN}$. In addition to the enhanced extraction of light from the LEDs, incorporation of PCs can be used to control and enhance the modulation speed of the LEDs. A slight narrowing of the far field emission pattern was observed in PC-LEDs.

The authors would like to acknowledge support by grants from DARPA, ARO, DOE, and NSF.

${ }^{1}$ S. X. Jin, J. Shakya, J. Y. Lin, and H. X. Jiang, Appl. Phys. Lett. 78, 3532 (2001).

${ }^{2}$ C. H. Chen, H. Hargis, J. M Woodall, and M. R. Melloch, Appl. Phys. Lett. 74, 3140 (1999).

${ }^{3}$ T. N. Order, J. Shakya, J. Y. Lin, and H. X. Jian, Appl. Phys. Lett. 83, 1231 (2003).

${ }^{4}$ T. N. Order, K. H. Kim, J. Y. Lin, and H. X. Jiang, Appl. Phys. Lett. 84, 466 (2004)

${ }^{5}$ J. Shakya, K. H. Kim, J. Y. Lin, and H. X. Jiang, Appl. Phys. Lett. 84, xxx (2004).

${ }^{6}$ G. Mayer, B. E. Malle, R. German, A. Forchel, P. Grambow, and H. P. Meier, Appl. Phys. Lett. 56, 2016 (1990).

${ }^{7}$ M. Boroditsky, I. Gontijo, M. Jackson, R. Vrijen, E. Yablonovitch, T. Krauss, C. C. Cheng, A. Schere, R. Bhat, and M. Krames, J. Appl. Phys. 87, 3497 (2000).

${ }^{8}$ V. Swaminathan, J. M. Freund, L. M. F. Chirovsky, T. D. Harris, N. A. Kuebler, and L. A. D' Asaro, J. Appl. Phys. 68, 4116 (1990).

${ }^{9}$ L. L. Chao, G. S. Cargill III, M. Levy, R. M. Osgood, Jr., and G. F. McLane, Appl. Phys. Lett. 70, 408 (1997).

${ }^{10}$ M. C. Beard, G. M. Turner, and C. A. Schmuttenmaer, Phys. Rev. B 62, 15764 (2000).

${ }^{11}$ http://www.phys.ksu.edu/area/GaNgroup/.

${ }^{12}$ P. E. Dodd, T. B. Stellwag, M. R. Melloch, and M. S. Lundstrom, IEEE Trans. Electron Devices 38, 1253 (1991).

${ }^{13}$ J. Li, K. B. Nam, K. H. Kim, J. Y. Lin, and H. X. Jiang, Appl. Phys. Lett. 78, 61 (2001).

${ }^{14}$ R. Aleksiejunas, M. Sudzius, T. Malinauskas, J. Vaitkus, K. Jarasiunas, and S. Sakai, Appl. Phys. Lett. 83, 1157 (2003). 